\title{
On the Strain-Induced Stabilization of Microstructural Features Formed Along Dislocations
}

\author{
M. H. Jhona, D. C. Chrzanb,c, and A. M. Glaeser ${ }^{b}$ \\ a Institute of High Performance Computing, A*STAR, Singapore 138632 \\ b Department of Materials Science and Engineering, University of California, Berkeley, \\ California 94720, USA \\ c Materials Sciences Division, Lawrence Berkeley National Laboratory, Berkeley, California \\ 94720, USA
}

Capillarity-driven mass transport limits the stability of microstructures with a high surface-tovolume ratio. Fiber reinforcements, dendritic structures, and other wire-like morphologies may be susceptible to Rayleigh instabilities and to concurrent or subsequent coarsening. Decreases in the characteristic length scales of microstructures to the nanoscale make such forms of evolution and instability evident at lower values of homologous temperature, consistent with expectations based on size scaling. Herein, we present a simple continuum theory that predicts that sufficiently small second-phase wires exhibiting dislocation character are stable to both Rayleigh instabilities and coarsening. Thus, defects such as hollow-core dislocations will tend to be stabilized while a freestanding nanowire will tend to be unstable. More generally, the effects of surface-energy anisotropy and strain energy on morphological stability are evaluated in a manner that allows their individual and combined effects on stability to be assessed and mapped.

Keywords: Rayleigh instabilities, coarsening, surface/interfacial energy anisotropy, strain energy, (hollow-core) dislocation 


\section{Introduction}

Modern engineering materials often contain high-aspect-ratio features such as eutectic rod-like microstructures. The utility of these assemblies can be limited at elevated temperatures because of the multifold instability of rod-like structures. Rayleigh instabilities and coarsening are examples of capillarity-driven modes of microstructural instability; both cause morphological changes with potentially deleterious effects on material properties. They reduce the excess interfacial energy per unit volume, and decrease the interface-to-volume ratio in isotropic systems.

The Rayleigh instability, sometimes referred to as pearling or fragmentation, refers to the susceptibility of an individual rod to the growth of periodic axial perturbations having a wavelength exceeding a critical minimum value, as shown in Fig. 1. This process will eventually lead to rod breakup into an array of equiaxed structures [1]. Coarsening, or Ostwald ripening, refers to a process in which a dispersion of particles or precipitates progressively increases its mean size through diffusional interactions. This process can be interpreted as the growth of larger particles at the expense of the smaller ones.

These two modes of microstructural evolution become more pronounced for smaller features. Based on scaling-law arguments for self-similar morphological changes through surface and interface diffusion [2], the time required for Rayleigh breakup is proportional to $R_{0}^{4}$, where $R_{0}$ is the unperturbed rod radius. Thus, a tenfold decrease in $R_{0}$ is expected to reduce the breakup time by a factor of $10^{4}$. Likewise, the coarsening rate increases as the mean precipitate or particle size decreases. Again assuming self-similarity, the mean rod radius increases with time $t$ at fixed temperature, and is proportional to $t^{1 / 3}$ or $t^{1 / 4}$, depending upon the dominant growth mechanism [3]. In other words, the rate of change is highest at short times when the average size is smallest.

The predicted time and size dependencies for coarsening and Rayleigh breakup indicate that stabilization of the fine-scale structures of current interest will pose tremendous challenges that will become increasingly severe as dimensional scales are 
further reduced. This has already been observed in a variety of nm-scale systems. For example, studies of the morphological evolution of Au nanowires with $R_{0}=12.5-66 \mathrm{~nm}$ show that breakup can occur in $<30 \mathrm{~min}$ at $300^{\circ} \mathrm{C}$ for the finest-size fibers [4]. As $R_{0}$ is decreased, breakup occurs in less time at fixed temperature, and at lower temperature for fixed anneal time. Similar trends are suggested by studies of $\mathrm{Cu}$ nanowires ranging from 30 $\mathrm{nm}$ to $120 \mathrm{~nm}$ in diameter; fragmentation of 40-nm diameter wires occurs in as little as 20 $\min$ at $400^{\circ} \mathrm{C}[5]$. For $\mathrm{Ag}$, "10-nm in diameter" nanobelts undergo fragmentation at room temperature. Kolb et al. [6] have shown that $\mathrm{Si}$-core-SiO${ }_{2}$-shell wires with diameters $>100$ $\mathrm{nm}$ develop periodic variations in the inner $(\mathrm{Si})$ and outer $\left(\mathrm{SiO}_{2}\right)$ radii, and breakup of the Si core can occur in the $900-1000^{\circ} \mathrm{C}$ range in times as short as $2 \mathrm{~h}$. These reported instances of fiber instability or nanowire "decomposition" may reflect Rayleigh instabilities, and additional examples are likely to emerge.

Prior work has shown that the presence of surface-energy anisotropy can delay breakup and stabilize fibers and internal rod-like voids [7]. The present work considers the ability of strain energy to stabilize nanowires and nanowire arrays, and the combined effects of strain energy and surface-energy anisotropy are explored. Specifically, we examine the consequences of the strain field of a dislocation on both the coarsening and Rayleigh instability of single-crystal nanowires grown along dislocations. The analysis is also relevant to the rod-like voids known as hollow-core dislocations, as found in GaN [8] and $\mathrm{SiC}$ [9]. We show that strain energy alone can fully suppress both coarsening and the Rayleigh instability when $R_{0}$ is sufficiently small, and that elastic strain energy and surface or interfacial-energy anisotropy working in tandem can delay breakup significantly at larger $R_{0}$. This suggests strategies for fabricating nanowire arrays suited for use at elevated temperatures where diffusion is active.

\section{Background}

Various strategies have been developed to stabilize microstructures with a high 
interface-area-to-volume ratio, typically by modifying the driving force for microstructural change. For example, the initial particle-size distribution of a powder can be narrowed, generating very fine grain size by reducing the driving force for coarsening [10]. In systems such as age-hardenable Ni alloys, the precipitate/matrix interfacial energy is low, reducing the interfacial-energy component of the driving force for coarsening [11]. Similarly, decreasing the solubility of the dispersed phase in the surrounding matrix or the diffusivity of the precipitate constituent decreases the solubility-diffusivity product and thus the chemical driving force for microstructural change [12].

A particularly important strategy for stabilizing high-aspect-ratio structures is to carefully choose the system crystallography to exploit surface-energy anisotropy. Cahn [13] first formulated this effect in the context of the Rayleigh breakup of a rod with anisotropic surface energy. The analysis assumes that the surface energy is radially isotropic in sections normal to the rod axis, resulting in a circular cross section. Anisotropy arises when axial variations in the radius incline the surface by an angle $\phi=\partial R / \partial z$ to the rod axis. Surface-energy anisotropy significantly alters rod breakup. In systems with isotropic surface energy, the rod is unstable to fluctuations with $\lambda>\lambda_{\min }^{\text {iso }}=2 \pi R_{0}$. However, when the orientation of the unperturbed surface lies in a local surface-energy minimum, the minimum wavelength for instability, $\lambda_{\min }$ is increased relative to $\lambda_{\min }^{\text {iso }}$. When the unperturbed surface lies in a cusp orientation, the rod is stabilized against all infinitesimal perturbations. Stölken and Glaeser [14] assessed the impact of such surface-energyanisotropy-induced stabilization on surface- and interface diffusion-controlled evolution, and showed that the kinetically dominant wavelength, $\lambda_{\max }$, is also shifted, but the ratio of $\lambda_{\max } / \lambda_{\min }$ remains $\sqrt{2}$, unchanged from that of isotropic systems. Surface-energy anisotropy strongly affects the kinetics of breakup: each factor of $n$ increase in $\lambda_{\min }$ is expected to increase the breakup time by a factor of $\mathrm{n}^{2}$. More recent contributions to this topic have addressed more complex forms of the surface-energy anisotropy, including transverse anisotropy [15]. 
Strain energy can likewise modify the driving force for capillarity-driven processes. For coarsening processes, strain interactions have been implicated as the basis for the development of aligned, or periodic arrays of discrete precipitates in bulk alloys, and conditions have been formulated that would lead to inverse coarsening, a narrowing of the size distribution and the development of stable monosized arrays of particles [16]. Sridhar et al. [17] found that misfit strains may alter the wavelengths for which a rod is susceptible to the Rayleigh instability. However, misfit strains only reduce the driving force for Rayleigh breakup; they cannot completely stabilize a wire. On the other hand, the strain energy of a dislocation has been predicted to stabilize high-aspect-ratio structures. Frank [18] proposed that if the surface energy is sufficiently low, the cores of dislocations could open to form a hollow-core dislocation or "micropipe" as a means of reducing the elasticstrain energy of a system. Such hollow-core structures have been observed experimentally in a number of systems, including GaN [19], SiC [20], and AlN [21]. These structures are unusual because they are voids that exhibit the strain field associated with dislocations. Their shape is determined by balancing the strain-energy reduction of an open core with the energy penalty for creating new surfaces. Their equilibrium radius was estimated to be $R_{f}=A / 2 \pi \gamma_{s v}$, where $\mathrm{A}$ is $G b^{2} / 4 \pi(1-v)$ for an edge dislocation and $G b^{2} / 4 \pi$ for a screw dislocation, $\mathrm{G}$ the shear modulus, $\mathrm{b}$ the length of the Burgers vector, Poisson's ratio, and $\gamma_{s v}$ is the solid-vapor interfacial energy. However, the theory of Frank [18] was not able to quantitatively predict the sizes of experimentally observed hollow-core dislocations.

Other authors have suggested refinements to this model. For instance, the stability of these defects has been considered when the crystal is growing or evaporating [22]. In other studies, the non-linear elastic behavior of the dislocation core was incorporated into estimates of the strain energy [23-25]. Alternatively, observations of oxygen segregation to hollow-core dislocations in GaN led to a proposed mechanism involving oxygen diffusion to surface pits [26]. Pirouz [19] proposed a mechanism based on dislocation attraction to triple junctions to resolve inconsistencies between the character of observed hollow-core 
dislocations and normal closed-core dislocations. Hollow-core dislocations may be formed because of kinetic processes, and are not necessarily microstructurally stable. Likewise, the strain energy of a dislocation is known to play a key role in heterogeneous nucleation. Cahn [27] noted that by forming a precipitate along a dislocation, the strain energy decrease augments the chemical driving force and can lead to either barrier-free nucleation and growth or to the development of a metastable precipitate whose size reflects a balance between the strain- and chemical-energy reductions and surface-energy increase accompanying nuclei growth. Nakamura et al. [28] have reported an approach to growing nanowires along dislocation cores in sapphire.

\section{Analysis}

For brevity, we refer to all rod-like microstructural features as wires in this analysis. However, it should be understood that the analysis applies to both cylindrical precipitates formed on dislocations and hollow-core dislocations. We model these two features as structures that exhibit a dislocation character, where they generate a long-range stress field in the surrounding matrix. However, for both situations, the strain energy inside the rod-like structure vanishes. For a second-phase particle, we follow Cahn and assume that the interface is incoherent, while for a hollow-core dislocation, the feature is a void that cannot support strain. The analysis of wire breakup is presented in three parts. First, we consider the effect of surface-energy anisotropy and strain energy on the minimum thermodynamically allowed wavelength, $\lambda_{\min }$. Second, we assess the kinetics of evolution defining the initially fastest-growing wavelength, $\lambda_{\max }$. The expressions derived in these two sections are general and applicable to a wide range of systems, including those without a dislocation. In the third section, we calculate the driving force for coarsening in an ensemble of rod-like precipitates.

3.1 The minimum thermodynamically allowed wavelength for Rayleigh instability, $\min$ The Rayleigh instability does not occur for all perturbation wavelengths. Only 
wavelengths larger than $\lambda_{\min }$ will be unstable. To calculate $\lambda_{\min }$ for wires with dislocation character, it is first necessary to develop an expression for the wire free energy. Following Frank [18] and Cahn [27], we consider only axisymmetric shapes, and estimate the free energy of a nearly cylindrical wire of radius $R_{0}$ with a dislocation character to be

$$
F[R(z)]=\int_{0}^{\lambda}\left[2 \pi R \gamma \sqrt{1+(\partial R / \partial z)^{2}} d z-A \log R-\pi R^{2} f\right] d z
$$

where $-\mathrm{f}$ is the volumetric free energy of formation of the wire phase, and is the energy per unit area of the wire-matrix interface. The free energy change stemming from a perturbation of wavelength,$\Delta F=F[R($ perturbed $)]-F[R($ unperturbed $)]$, is calculated with Eqn. (1). For volume-conserving perturbations of the form $R(z)=R_{1}+\delta \cos (2 \pi z / \lambda)$ with small relative to $R_{0}, R_{0}$ and $R_{1}$ are related by $R_{0}^{2}-R_{1}^{2}=\delta^{2} / 2$. Following Cahn [13], we take the interfacial energy to be anisotropic, with ( ) assumed to be a function only of $\phi=\partial R / \partial z$. We also assume that ( ) may be expanded about $\phi=0$ as

$$
\gamma(\phi)=\gamma_{0}+\phi\left(\frac{\partial \gamma}{\partial \phi}\right)_{\phi=0}+\frac{1}{2} \phi^{2}\left(\frac{\partial^{2} \gamma}{\partial \phi^{2}}\right)_{\phi=0}+O\left(\phi^{3}\right)
$$

Retaining terms to order ${ }^{2}$, one obtains

$$
\Delta F=\left[\frac{A \lambda^{2}}{2 R_{0}^{2}}-\frac{\pi \gamma \lambda}{2 R_{0}}+\frac{2 \pi^{3} R_{0}}{\lambda}\left[\gamma_{0}+\left(\frac{\partial^{2} \gamma}{\partial \phi^{2}}\right)_{\phi=0}\right]\right] \delta^{2}
$$

A wire will be unstable to perturbations for which $\Delta F<0$, when exceeds a critical value,

$$
\lambda>2 \pi R_{0} \sqrt{\frac{1+\gamma_{0}^{-1}\left(\partial^{2} \gamma / \partial \phi^{2}\right)_{\phi=0}}{1-\left(2 R^{*} / R_{0}\right)}}
$$

where we define a Frank radius $R^{*}=A / 2 \pi \gamma_{0}$, with respect to the $\phi=0$ interface energy. Paralleling comparisons of homogeneous and heterogeneous nucleation, the critical condition on can be rewritten as a product of the critical or minimum wavelength in an isotropic system, $\lambda_{\min }^{\text {iso }}$, and a stabilization factor, $\mathrm{S}$, that includes contributions from both surface-energy anisotropy and elastic-strain energy, i.e., $\lambda_{\min }=S \lambda_{\min }^{\text {iso }}$. For a system with no 
dislocation, $R^{*}=0$ and the result then duplicates the earlier finding of Cahn [13]. Whether surface-energy anisotropy increases or decreases $\lambda_{\min }$, or equivalently whether $\mathrm{S}>1$ or $\mathrm{S}$ $<1$, hinges solely upon the sign of $\partial^{2} \gamma / \partial \phi^{2}$. In sapphire, experiments investigating the morphological evolution of high-aspect-ratio rod-like pores suggest that for some orientations, $S$ may exceed 10 even when $R^{*}=0$. For a system in which is isotropic, the numerator of S equals unity, and the degree of stabilization then hinges solely on the ratio $R_{0} / R^{*}$. The wire will be completely stable to perturbations if $R_{0} \leq 2 R^{*}$, and thus, dislocation pipes and nanowires in this size range are immune to Rayleigh instability. S drops off rapidly once $R_{0}$ exceeds $2 R^{*}$; when $R_{0}=4 R^{*}, S=2$. A plot of $S$ versus $R_{0} / R^{*}$ for an isotropic system, Fig. 2, shows this drop-off for values of $R_{0}>2 R^{*}$. When both elasticstrain energy and surface-energy anisotropy are active, then it is in this range of $R_{0}>2 R^{*}$ that the two factors can either reinforce one another to increase stability, or oppose one another. A wide spectrum of behaviors is thus possible, including situations where $\lambda_{\min }<2 \pi R_{0}$. To illustrate this, we include two additional curves, one in which $1+\gamma_{0}^{-1}\left(\partial^{2} \gamma / \partial \phi^{2}\right)_{\phi=0}$ assumes a value of 4 , and a second in which it assumes a value of 0.25. When the bounding surface of the unperturbed cylinder lies in an energy minimum, then the range of $R_{0} / R^{*}$ in which strong stabilization occurs is expanded. When instead the bounding surface of the unperturbed cylinder lies in an energy maximum, then the stabilizing effects of strain energy are rapidly overwhelmed and perturbations with $\lambda<2 \pi R_{0}$ are possible over a wide range of $R_{0} / R^{*}$ values.

3.2 The kinetically dominant wavelength for Rayleigh instability, $\lambda_{\max }$

The wavelength of the initially fastest-growing perturbation, $\lambda_{\max }$, reflects the relevant active mass-transport mechanisms. Rayleigh [1] established that perturbations of different wavelengths grow at different rates: for inviscid fluid jets, their amplitude growth rate is maximized when $\lambda \approx 9.02 R_{0}$. An extension of the Rayleigh analysis by Nichols and Mullins showed that the fastest diverging wavelength is determined by the interplay 
between thermodynamic factors and the dominant mechanism of mass transport [29]. At sufficiently small sizes, surface or interfacial diffusion is more likely to be the dominant transport path. When a solid wire forms in a matrix, morphological evolution can also occur by the flow of atoms through the surrounding matrix (external volume diffusion) or flow of atoms within the rod (internal volume diffusion). Nichols and Mullins evaluated how different mass-transport mechanisms and transport paths affect $\lambda_{\max }$ for a rod with isotropic interface energy undergoing Rayleigh instability [29]. Stölken and Glaeser extended this work by assessing the influence of surface-energy anisotropy on the kinetics of surface- or interfacial-diffusion-controlled evolution of rod-like phases [14].

In this section, we extend these two studies to include systems that exhibit a dislocation character as well as anisotropic surface/interface energy where the transport mechanisms of surface/interfacial diffusion and volume diffusion external and internal to the rod-like phase are possible. While the volume-diffusion analysis may be of limited utility for nanowires, these solutions supplement the work of Stölken and Glaeser and provide a full set of kinetic expressions for the evolution of the model system proposed by Cahn [13].

\subsubsection{Surface/Interfacial-diffusion-controlled evolution}

In the isotropic system considered by Nichols and Mullins, the spatial variation of the surface chemical potential can be related to the surface Laplacian of the curvature, $\nabla_{s}^{2} \kappa$ . In the present system, with both strain energy and surface-energy anisotropy contributions, the definition of the chemical potential of the rods or wires constituent is more complex. Taking the first variation of Eqn. (1), and letting represent the atomic volume, one obtains

$$
\mu=\Omega \frac{\partial F}{\partial V}=\Omega \frac{\partial F}{\partial R} \frac{\partial R}{\partial V}=\frac{\Omega \gamma_{0}}{R}-\Omega\left[\gamma_{0}+\left(\frac{\partial^{2} \gamma}{\partial \phi^{2}}\right)_{\phi=0}\right] \frac{\partial^{2} R}{\partial z^{2}}-\frac{\Omega A}{2 \pi R^{2}}-\Omega f
$$

In isotropic systems, when shape changes are occurring by surface diffusion, Mullins has 
shown that the outward normal velocity at any point, $\dot{n}=(\partial n / \partial t)$, is related to the product of a constant $B$, and the surface Laplacian of the curvature, $\nabla_{s}^{2} \kappa$ [30]. In this more general case,

$$
\dot{n}=B \nabla_{s}^{2}\left[\frac{\gamma_{0}}{R}-\left(\gamma_{0}+\frac{\partial \gamma}{\partial \phi}\right) \frac{\partial R}{\partial z^{2}}-\gamma_{0} \frac{R^{*}}{R^{2}}\right]
$$

For small perturbations, $\dot{n}=\dot{R}$, and s, which is measured along the surface, is approximately equal to z. Following the method of Nichols and Mullins, we obtain an expression for the time-dependence of the amplitude of the form

$$
\left(\frac{\dot{\delta}}{\delta}\right)_{\text {surface }}=\frac{4 \pi^{2} B}{\lambda^{2}}\left[\frac{1}{R_{0}^{2}} \gamma_{0}-\frac{4 \pi B}{\lambda^{2}}\left[\gamma_{0}+\left(\frac{\partial^{2} \gamma}{\partial \phi^{2}}\right)_{\phi=0}\right]\right]-\gamma_{0} \frac{2 R^{*}}{R_{0}^{3}}
$$

where the dot represents the time derivative. Note that in contrast to the situation for isotropic and anisotropic strain-free model systems, the introduction of a strain-energy term causes a departure from a simple size-scaling relationship for the evolution time. This is true both for the present case of surface diffusion as well as the volume-diffusion situations discussed hereafter. Setting $\dot{\delta}$ equal to zero and solving for recovers Eqns. (4) and (5). Taking the derivative with respect to one finds that $(\dot{\delta} / \delta)$ is maximized at a wavelength

$$
\lambda_{\max }=2 \pi R_{0} \sqrt{2} \sqrt{\frac{1+\left(\partial^{2} \gamma / \partial \phi^{2}\right)_{\phi=0}}{1-2 R^{*} / R_{0}}}=\sqrt{2}\left(\lambda_{\text {min }}^{\text {isotropic }} S\right)=\sqrt{2} \lambda_{\text {min }}
$$

It follows that when $R_{0}>2 R^{*}$ and evolution is surface or interfacial-diffusion-controlled, a $\sqrt{2}$ scaling exists between $\lambda_{\max }$ and $\lambda_{\min }$ regardless of the specific combination of strain energy and surface-energy anisotropy that is operative. The result derived previously by Stölken and Glaeser is a specific case of this more general result.

\subsubsection{Volume-diffusion-controlled evolution}

In Nichols and Mullins' treatment of isotropic systems, the Gibbs-Thomson equation was used to describe the deviation in the local solute concentration, $\Delta c$, from an 
equilibrium bulk value. In the isotropic system, the key parameter is the curvature. In the system under consideration here, where strain-energy and surface-energy-anisotropy contributions can arise, it is necessary to develop an expression that includes both effects. A modified version of the Gibbs-Thomson equation can be derived, and is used here to extend the original treatments of Nichols and Mullins to a rod (or pipe) grown along a dislocation.

When effects of a dislocation's strain energy and of surface-energy (or interfacialenergy) anisotropy are considered, the Gibbs-Thomson equation takes the form

$$
\Delta c=\frac{c_{0} \Omega}{k_{B} T}\left[\frac{\gamma_{0}}{R}-\left[\gamma_{0}+\left(\frac{\partial^{2} \gamma}{\partial \phi^{2}}\right)_{\phi=0}\right] \frac{\partial^{2} R}{\partial z^{2}}-\gamma_{0} \frac{R^{*}}{R^{2}}\right]
$$

where $c_{0}$ is the equilibrium concentration of solute atoms expressed as the number of solute atoms per unit volume at a flat interface, and $\Delta c=c-c_{0}$ is the deviation from this value due to curvature and anisotropy. Following the procedures and assumptions of Nichols and Mullins, when diffusion occurs in the volume external to the rod-like phase,

$$
\left(\frac{\dot{\delta}}{\delta}\right)_{\mathrm{int}}=\frac{D \Omega c_{0} \gamma_{0}}{k_{B} T}\left(1-\frac{2 R^{*}}{R_{0}}\right)\left[\frac{1}{R_{0}^{2}}-(S \omega)^{2}\right] \frac{K_{1}\left(\omega R_{0}\right)}{K_{0}\left(\omega R_{0}\right)}
$$

where $D$ is the bulk diffusion coefficient, is the atomic volume, $\omega=2 \pi / \lambda$, and $K_{n}$ is the $n$th modified Bessel function of the second kind. This expression would describe the volume-diffusion contribution to the evolution of rod-like voids. For volume diffusion within the rod, the relevant expression for $(\dot{\delta} / \delta)$ is

$$
\left(\frac{\dot{\delta}}{\delta}\right)_{\mathrm{int}}=\frac{D \Omega c_{0} \gamma_{0}}{k_{B} T}\left(1-\frac{2 R^{*}}{R_{0}}\right)\left[\frac{1}{R_{0}^{2}}-(S \omega)^{2}\right] \frac{I_{1}\left(\omega R_{0}\right)}{I_{0}\left(\omega R_{0}\right)}
$$

where $I_{n}$ is the $n$th modified Bessel function of the first kind.

\subsection{Comparison to systems with no dislocation character}

In many two-phase systems, rod-like pores and particles evolve into discrete pores and particles after some time at elevated temperature. In many of these cases, the rod-like 
phase is bounded by surfaces or interfaces with anisotropic energy, but does not necessarily have a dislocation character. $\lambda_{\max }$ is often related to the size and spacing of discrete pores and particles, and from this, the dominant transport mechanism has been inferred, assuming that the isotropic model is valid. When this is true, one expects $\lambda_{\max }$ will be $8.89 R_{0}$ for surface or interfacial diffusion, $9.02 R_{0}$ for internal volume diffusion, and $12.96 R_{0}$ for external volume diffusion. Additionally, in isotropic systems the ratios $\lambda_{\max }^{\text {int }} / \lambda_{\max }^{\text {surf }}$ and $\lambda_{\max }^{\text {ext }} / \lambda_{\max }^{\text {surf }}$ are expected to be 1.015 and 1.458 , respectively. Using the results of the present analysis, one can extend the analyses of Nichols and Mullins and assess how these ratios are affected by surface- or interfacial-energy anisotropy. The results are shown in Fig. 3.

In the three cases that we examined, our expression for $(\dot{\delta} / \delta)$ is altered from the classical analyses of Nichols and Mullins by including strain and surface-energy anisotropy terms. To better understand how the breakup dynamics are affected, we compare $(\dot{\delta} / \delta)$ for an arbitrary system to that of an isotropic, dislocation-free system with $R^{*}=0$ and $S=1$

Eqns. (7) and (8) can be combined to yield:

$$
\left(\frac{\dot{\delta}}{\delta}\right)_{\text {surface }}=B \omega^{2}\left(1-\frac{2 R^{*}}{R_{0}}\right)\left[\frac{1}{R_{0}^{2}}-(S \omega)^{2}\right]
$$

Comparing this expression to the reference state, we find:

$$
\frac{(\dot{\delta} / \delta)_{\text {surface }}}{(\dot{\delta} / \delta)_{\text {surface }}^{\text {reference }}}=\frac{\dot{\delta}_{\text {surface }}}{\dot{\delta}_{\text {surface }}^{\text {referce }}}=\left(1-\frac{2 R^{*}}{R_{0}}\right)\left[\frac{1-\left(\lambda_{\min } / \lambda\right)^{2}}{1-\left(\lambda_{\min }^{\text {referce }} / \lambda\right)^{2}}\right]
$$

As $R_{0}$ decreases and approaches $2 R^{*}, \dot{\delta}_{\text {surface }}$ goes to zero. In addition, when $S>1$, $\lambda_{\min }>\lambda_{\min }^{\text {isotropic }}=2 \pi R_{0}$, further reducing $\dot{\delta}_{\text {surface }}$ relative to that in the isotropic, dislocation-free model system when $\lambda>\lambda_{\min }$. We may likewise normalize our expressions for $(\dot{\delta} / \delta)$ for external volume diffusion and internal volume diffusion to obtain the rather surprising result, 


$$
\frac{(\dot{\delta} / \delta)_{\text {surface }}}{(\dot{\delta} / \delta)_{\text {surface }}^{\text {reference }}}=\frac{(\dot{\delta} / \delta)_{\text {ext }}}{(\dot{\delta} / \delta)_{\text {ext }}^{\text {reference }}}=\frac{(\dot{\delta} / \delta)_{\text {int }}}{(\dot{\delta} / \delta)_{\text {int }}^{\text {reference }}}
$$

A plot of this normalized amplitude growth rate, valid for all three mechanisms, is presented in Fig. 4.

\subsection{Coarsening of Nanowires}

In the previous sections, the presence of a dislocation and its associated strain energy were shown to affect the energetics of axial instabilities and thus the Rayleigh wavelength. The energy changes accompanying changes in the nanowire radius will also alter the chemical potential gradients that drive other capillary-driven instabilities. In particular, the driving forces that govern coarsening are altered. To illustrate this, consider an ensemble of parallel, cylindrical wires that can exchange mass with each other. The chemical potential of the th wire will determine whether or not the wire will grow. Denoting the radius of the $i$ th wire as $R_{i}$, the chemical potential of the $i$ th wire is

$$
\mu_{i}=-\frac{A \Omega}{2 \pi R_{i}^{2}}+\frac{\gamma \Omega}{R_{i}^{2}}=\frac{\gamma \Omega}{R^{*}}\left(-\frac{R^{* 2}}{R_{i}^{2}}+\frac{R^{*}}{R_{i}}\right)
$$

The chemical potential $\mu_{i}$ has a maximum at $R_{i}=2 R^{*}$, as illustrated in Fig. 5. Under the mean-field approximation, the growth rate of the th wire is driven by the chemical potential difference $\Delta \mu=\bar{\mu}-\mu_{i}$, where the system average chemical potential is given by $\bar{\mu}=\sum \mu_{i} / n$. To interpret this result, first consider the case in which the largest wire is smaller than $2 R^{*}$. In that case, the chemical potential of the larger wire will decrease as it shrinks. If the total volume of the cylinders is conserved, then equilibrium can be reached if the smaller wire grows and the larger wire shrinks, thus causing inverse coarsening. However, if the largest wire is larger than $2 R^{*}$, then its chemical potential will decrease when it grows. It should then tend to grow at the expense of the smaller wire. This result can be contrasted with the classical work of Frank [18]. While Frank predicts that a hollowcore dislocation of radius $R^{*}$ is thermodynamically stable, our coarsening analysis predicts that dislocations or rods smaller than radius $2 R^{*}$ can be stable, depending on the size of the 
largest rod in the distribution.

4. Discussion

There are four major approximations made in the current analysis. First, the elastic boundary condition at the interface between the wire and the matrix is highly simplified. Second, the linear stability analysis is only valid very close to the point of instability. Third, we consider only axisymmetric shapes. Finally, we do not consider the effects of surfaceenergy anisotropy on the kinetics of morphological evolution. Herring was the first to discuss the effects of faceting on surface movement as would occur during sintering and during shape-equilibration processes. Mullins and Rohrer considered the specific problem of a partially faceted particle or cavity approaching an equilibrium or Wulff shape, and showed that the need to nucleate an atomic-height patch of atoms or an atomic depth cavity on migrating facets introduced a nucleation energy barrier that could effectively lock a particle or cavity into a nonequilibrium shape [31]. Examples of this are provided by the in situ TEM experiments of Dahmen et al. examining lead inclusions in aluminum [32], and studies of the shape evolution of lithographically defined coin-shaped pores in sapphire [33] and SiC [34]. In the case of pores, changes in the crystallographic orientation of geometrically identical flaws led to substantial differences in the evolution rates; pores initially bounded by stable surfaces evolve at rates orders of magnitude lower than those bounded by unstable surfaces.

However, even though the current theory is somewhat simplified, it provides an intuitive way to interpret many existing experiments. The anisotropy factor S summarizes the competition between driving forces for structural change present in these systems: strain energy and surface-energy anisotropy. We can validate the conclusions of our linear stability analysis by considering observations of hollow-core dislocations as well as nanowires formed on dislocations.

Hollow-core dislocations have been observed in several systems, including $\mathrm{SiC}, \mathrm{GaN}$, and AlN films. The Frank radius of hollow-core dislocations in GaN is about $1 \mathrm{~nm}$. Observed 
dislocation pipe radii in GaN are consistently larger than $1 \mathrm{~nm}$; values of $3.530 \mathrm{~nm}$ were reported by Qian et al. [35] for nearly circular pipes along the [0001] direction, a broader range from 3.5 to $500 \mathrm{~nm}$ has been indicated in some studies, and radii as large as $1500 \mathrm{~nm}$ have been cited. This wide range of hollow-core dislocation sizes has been attributed to modification of the surface energy by impurities and kinetic effects due of the growth process [19]. Such hollow-core dislocations may be larger than the Frank radius, and therefore may undergo microstructural change.

Of particular importance to our study is the observation that hollow-core dislocations may contain alternating open and closed segments in GaN films. These chains of voids along dislocations in these films may be either precursors to a nanopipe, or the consequence of nanopipe instability. Pailloux et al. [36] have examined the morphologic evolution of [0001] nanopipes in GaN with initial radii of $\sim 5 \mathrm{~nm}$ during TEM observation (electron radiation). The breakup of the structures involved the growth of periodic perturbations, qualitatively consistent with the predictions of the present analysis. Reported decomposition wavelengths were in the range of $1050 \mathrm{~nm}$, and thus much greater than $\lambda_{\max }$ in an isotropic system. Although strain energy tends to increase $\lambda_{\min }$, since $R_{0} \approx 5 R^{*}$, the strain-energy contribution to stabilization would be small as indicated in Fig. 2, and the observed long wavelengths must be attributed primarily to surface-energy anisotropy.

Ikuhara [37] recently reviewed the subject of "nanowires" grown on dislocations. It is uncertain whether the wires exist as a discrete second phase, or as a solute cloud surrounding the dislocation core. The Ti-based nanowires grown along dislocations in sapphire, as described by Nakamura et al. [28] have an estimated radius of $2.5 \mathrm{~nm}$ and extend through a roughly $10-\mu \mathrm{m}$ thick sapphire substrate. It is certain that in the absence of any stabilizing effects, second-phase wires of this aspect ratio and radius would be prone to break up. For a second-phase wire grown on a dislocation in sapphire, using the following literature values for sapphire, $\mathrm{G}=150 \mathrm{GPa}, v=0.24$, and $b=0.476 \mathrm{~nm}$, and taking the 
interfacial energy to be $\sim 1.0 \mathrm{~J} / \mathrm{m}^{2}$ one estimates that $2 R^{*}$ is $\approx 1.13 \mathrm{~nm}$. Thus, stabilizing a $2.5 \mathrm{~nm}$ radius second-phase wire would likely require a significant additional contribution from surface-energy anisotropy. Comparisons of Wulff shapes of voids in sapphire and those of Pt nanoparticles embedded in sapphire [38] show an overlap between the crystal planes in the Wulff shape of sapphire and those on the sapphire side of Pt/sapphire interfaces in equilibrated Pt nanoparticles. In Nakamura's study, the dislocations and the corresponding nanowires are oriented along the [1ㅣㅣㅇㅣ direction of sapphire. If secondphase wires could be grown along these dislocations, the potential exists for low-index bounding facets of the $\mathbf{c}(0001), \mathbf{a}\{11 \overline{2} 0\}$ and $\mathbf{p}\{11 \overline{2} 3\}$ types, all of which are present in the Wulff shape of undoped sapphire to form, and assist in kinetic stabilization.

The central feature of our analysis is that nanometer-scale microstructural features can be stabilized if the feature itself has a dislocation character. This is not the case for structures such as a freestanding whisker with an axial dislocation. As found by Kirill et al., the strain from a dislocation tends to destabilize a whisker and can further lead to asymmetric breakup modes [39]. Because the rod itself (instead of the matrix) is strained, fluctuations will tend to reduce the strain energy, lowering the minimum wavelength for breakup. According to our linear stability analysis, a whisker will break up with a minimum wavelength $\lambda_{\min }^{\text {whisker }}=2 \pi R_{0} \sqrt{\left(1+\gamma^{\prime \prime} / \gamma_{0}\right) /\left(1+2 R^{*} / R_{0}\right)}$.

5. Conclusions

We developed general dynamical equations that describe the evolution of axisymmetric, high-aspect ratio nanostructures. In particular, we considered both the effects of strain due to the dislocation character of the nanostructure, as well as surfaceenergy anisotropy. Linear stability analysis of these dynamical equations demonstrates that an initially cylindrical precipitate embedded in a solid matrix can be thermodynamically stabilized if it has a dislocation character. This includes experimentally observed defects such as hollow-core dislocations. We found that cylindrical rods with isotropic interface energy will be stable to morphological change if their radii are smaller 
than twice the Frank radius. Anisotropic interface energies can serve to either stabilize or destabilize a structure, depending on the sign of $2 / 2$.

Acknowledgements

Work by DCC was supported by the U.S. Department of Energy (DOE), Office of Science, Basic Energy Sciences (BES) under Award \#DE-AC02- 05CH11231. AMG acknowledges long-term support from the GRF. 


\section{References}

[1] L. Rayleigh, On the instability of jets, Lond. Math. Soc. Proc. 10 (1879) 4-13.

[2] C. Herring, Effect of change of scale on sintering phenomena, J. Appl. Phys. 21 (1950) 301-303.

[3] A.J. Ardell, Isotropic fiber coarsening in unidirectionally solidified eutectic alloys, Metall. Trans. 3 (1972) 1395-1401.

[4] S. Karim, M.E. Toimil-Molares, A.G. Balogh, W. Ensinger, T.W. Cornelius, E.U. Khan, R. Neumann, Morphological evolution of Au nanowires controlled by Rayleigh instability, Nanotechnol. 17 (2006) 5954-5959.

[5] M.E. Toimil-Molares, A.G. Balogh, T.W. Cornelius, R. Neumann, C. Trautmann, Fragmentation of nanowires driven by Rayleigh instability, Appl. Phys. Lett. 85 (2004) 5337-5339.

[6] F.M. Kolb, H. Hofmeister, M. Zacharias, U. Gösele, On the morphological instability of silicon/silicon dioxide nanowires, Appl. Phys. A 80 (2005) 1405-1408.

[7] M.K. Santala, A.M. Glaeser, Rayleigh instabilities in crystalline solids: Evolution of finiteaspect-ratio pore channels in sapphire, Acta Mater. 56 (2008) 1967-1980.

[8] D. Cherns, W.T. Young, J.W. Steeds, F.A. Ponce, S. Nakamura, Observation of coreless dislocations in $\alpha-G a N$, J. Cryst. Growth 178 (1997) 201-206.

[9] J. Heindl, H.P. Strunk, V.D. Heydemann, G. Pensl, Micropipes: Hollow tubes in silicon carbide, Phys. Stat. Sol. (a) 162 (1997) 251-262.

[10] Z. Fang, B. Patterson, Experimental investigation of particle-size distribution influence on diffusion-controlled coarsening, Acta Metall. Mater. 41 (1993) 2017-2024.

[11] A.J. Ardell, R.B. Nicholson, On the modulated structure of aged Ni-Al Alloys, Acta. Metall. 14 (1966) 1295-1309.

[12] A. Umantsev, G. Olson, Ostwald ripening in multicomponent alloys, Scripta Metall. Mater. 29 (1993) 1135.

[13] J.W. Cahn, Stability of rods with anisotropic surface energy, Scripta Met. 13 (1979) 1069-1071.

[14] J.S. Stölken and A.M. Glaeser, The morphological evolution of cylindrical rods with anisotropic surface free energy via surface diffusion, Scripta Metall. Mater. 27 (1992) 449454.

[15] K.F. Gurski, G.B. McFadden, The effect of anisotropic surface energy on the Rayleigh instability, Proc. Roy. Soc. A 459 (2003) 2575-2598.

[16] C.H. Su, P.W. Voorhees, The dynamics of precipitate evolution in elastically stressed solids-I. Inverse coarsening, Acta Mater. 44 (1996) 1987-1999.

[17] N. Sridhar, J.M. Rickman, D.J. Srolovitz, Microstructural stability of stressed lamellar and fiber composites, Acta Mater. 45 (1997) 2715-2733.

[18] F.C. Frank, Capillary equilibria of dislocated crystals, Acta Cryst. 4 (1951) 497-501.

[19] P. Pirouz, On micropipes and nanopipes in SiC and GaN, Phil. Mag. A 78 (1998) 727736.

[20] D. I. Cherednichenkoa, Y. I. Khlebnikov, I. I. Khlebnikov, R.V. Drachev, T.S. Sudarshan, Dislocations as a source of micropipe development in the growth of silicon carbide, J. Appl. Phys. 89 (2001) 4139-4141.

[21] Y. Tokumoto, N. Shibata, T. Mizoguchi, M. Sugiyama, Y. Shimogaki, J.-S. Yang, T. Yamamoto, Y. Ikuhara, High-resolution transmission electron microscopy (HRTEM) 
observation of dislocation structures in AlN thin films, Journal of Materials Research 23 (2008) 2188-2194.

[22] N. Cabrera, M. M. Levine, On the dislocation theory of evaporation of crystals, Phil. Mag. 1 (1956) 450-458.

[23] W. Schaarwächter, Zum mechanismus der versetzungsätzung I. Die bildung zweidimensionaler lochkeime an den enden von versetzungslinien, Phys. Stat. Solidi 12 (1965) 375-382.

[24] W. Schaarwächter, Zum mechanismus der versetzungsätzung II.

Entstehungsbedingungen für Ätzgruben, Phys. Stat. Solidi 12 (1965) 865-876.

[25] B. van der Hoek, J. van der Eerden, P. Bennema, Thermodynamical stability conditions for the occurrence of hollow cores caused by stress of line and planar defects, J. Crys. Growth 56 (1982) 621-632.

[26] M.E. Hawkridge D. Cherns, Oxygen segregation to dislocations in GaN, Appl. Phys. Lett. 87 (2005) 221903.

[27] J.W. Cahn, Nucleation on dislocations, Acta Metall. 5 (1957) 169-172.

[28] A. Nakamura, K. Matsunaga, J. Tohma, T. Yamamoto, Y. Ikuhara, Conducting nanowires in insulating ceramics, Nature Mat. 2 (2003) 453-456.

[29] F. A. Nichols, W. W. Mullins, Surface- (Interface-) and volume-diffusion contributions to morphological changes driven by capillarity, Trans. Metall. Soc. AIME 233 (1965) 18401848.

[30] W. W. Mullins, Theory of thermal grooving, J. Appl. Phys. 28 (1957) 333-339.

[31] G. S. Rohrer, C. L. Rohrer, W. W. Mullins, Nucleation energy barriers for volume conserving shape changes of crystals with nonequilibrium morphologies, J. Amer. Ceram. Soc. 84 (2001) 2099-2104.

[32] U. Dahmen, SQ. Xiao, S. Paciornik, E. Johnson, A. Johansen, Magic-size equilibrium shapes of nanoscale Pb inclusions of Al, Phys. Rev. Lett. 78 (1997) 471-474.

[33] M. Kitayama, T. Narushima, W. C. Carter, R. M. Cannon, A. M. Glaeser, The Wulff shape of alumina: I. Modeling the kinetics of morphological evolution, J. Amer. Ceram. Soc. 83 (2000) 2561-71.

[34] T. Narushima, A. M. Glaeser, High-temperature morphological evolution of lithographically introduced cavities in silicon carbide, J. Amer. Ceram. Soc. 84 (2001) 921928.

[35] W. Qian, G. S. Rohrer, M. Skowronski, K. Doverspike, L. Rowland, D. Gaskill, Open-core screw dislocations in GaN epilayers observed by scanning force microscopy and highresolution transmission electron microscopy, Appl. Phys. Lett. 67, 2284-2286 (1995). [36] F. Pailloux, J. Colin, J. F. Barbot, J. Grilhé, Pinch off of nanopipes under electron irradiation in GaN, Appl. Phys. Lett. 86 (2005) 131908.

[37] Y. Ikuhara, Nanowire design by dislocation technology, Prog. Mater. Sci. 54 (2009) 770-791.

[38] M. K. Santala, V. R. Radmilovic, R. Giulian, M. C. Ridgway, R. Gronsky, A. M. Glaeser, The orientation and morphology of platinum precipitates in sapphire, Acta Mater. 59 (2011) 4761-4774.

[39] D. J. Kirill, S. H. Davis, M. J. Miksis, P. W. Voorhees, Morphological instability of a whisker, Proc. Royal Soc. A 4555 (1999) 3825-3844. 


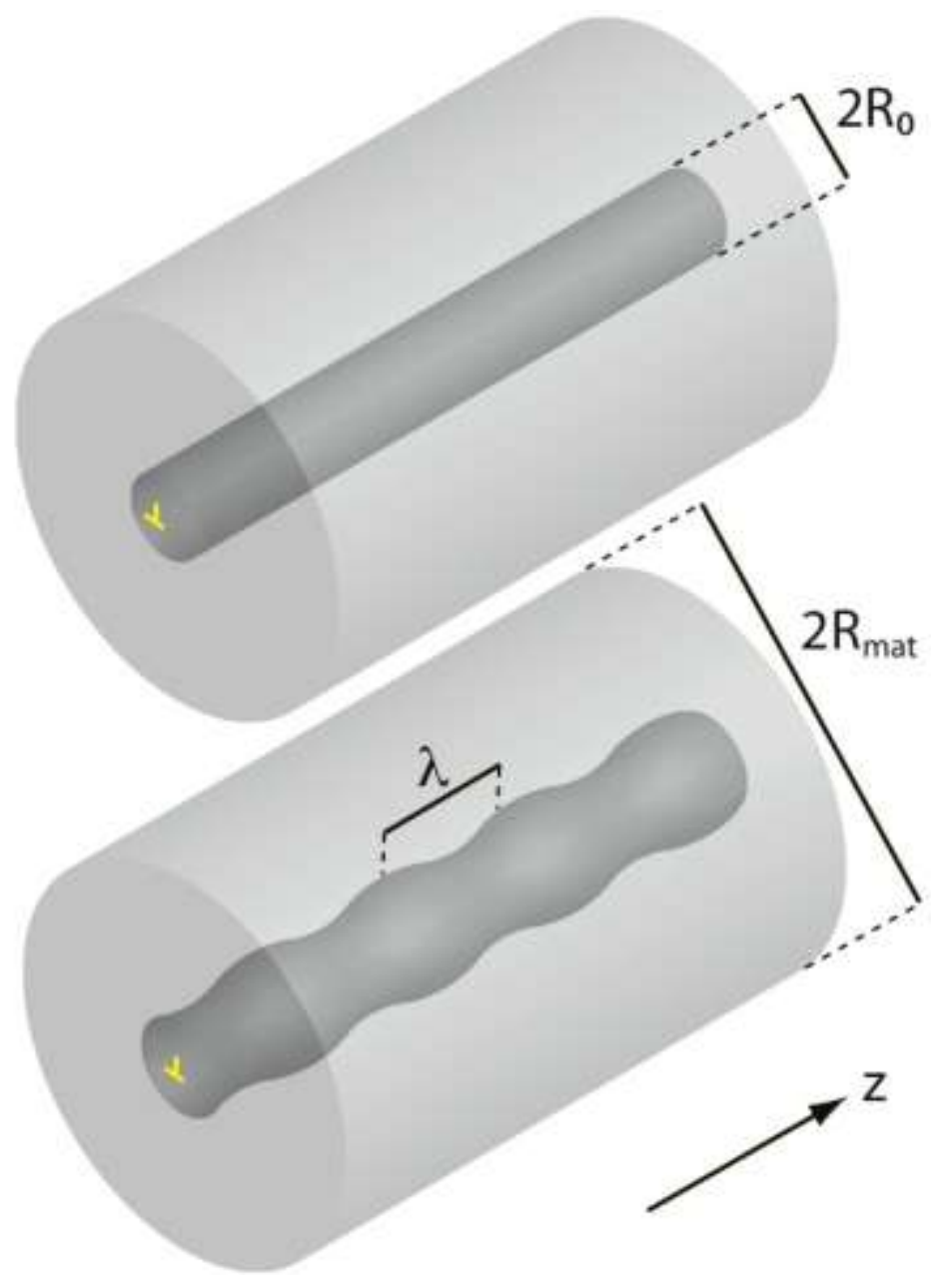

Fig. 1: Schematic illustration of a rod-like particle grown on a dislocation line and undergoing a Rayleigh instability with a characteristic wavelength . The top panel depicts the rod prior to the development of a discernible perturbation, while the lower panel shows the rod after the perturbation is well developed. The light grey region represents the matrix phase and the dark grey the precipitate phase. The dislocation line extends parallel to the axis of the cylinder. 


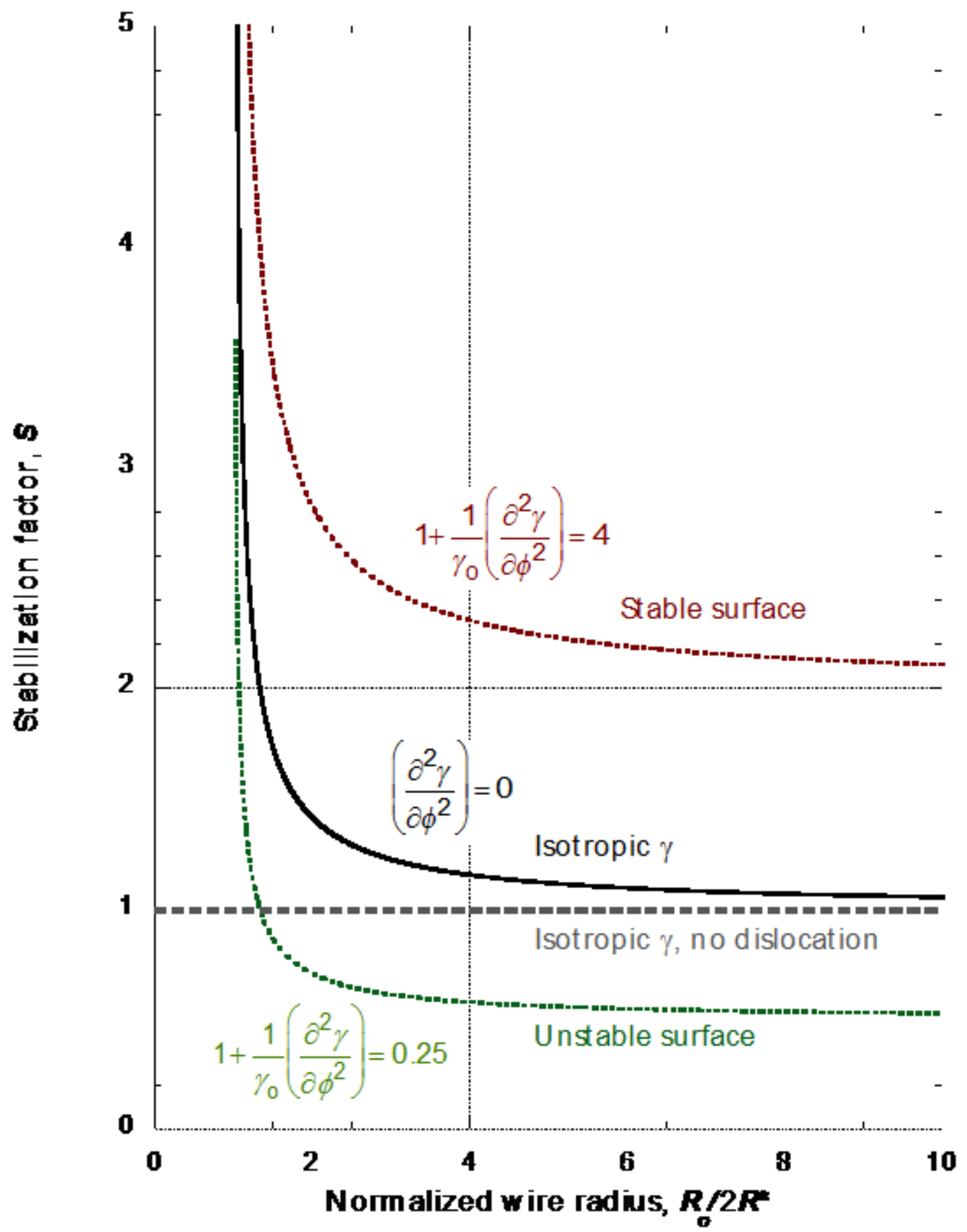

Fig. 2: Plot illustrating the effects of strain energy, surface/interfacial-energy anisotropy, and normalized wire radius on the stability of a rod-like particle. The parameter $\mathrm{S}$ is the ratio of the minimum stable perturbation wavelength in the actual system to that in an isotropic dislocation-free reference system, $\lambda_{\min }^{\text {actual }} / \lambda_{\min }^{\text {ref }}$; for the reference system, $S=1$ for all $R_{0}$. If a dislocation is present, then $S \rightarrow \infty$ as $R_{0} \rightarrow 2 R^{*}$. The values of $S$ when $R_{0} / 2 R^{*}>1$ hinge on the sign and magnitude of the surface/interfacial-energy anisotropy. 


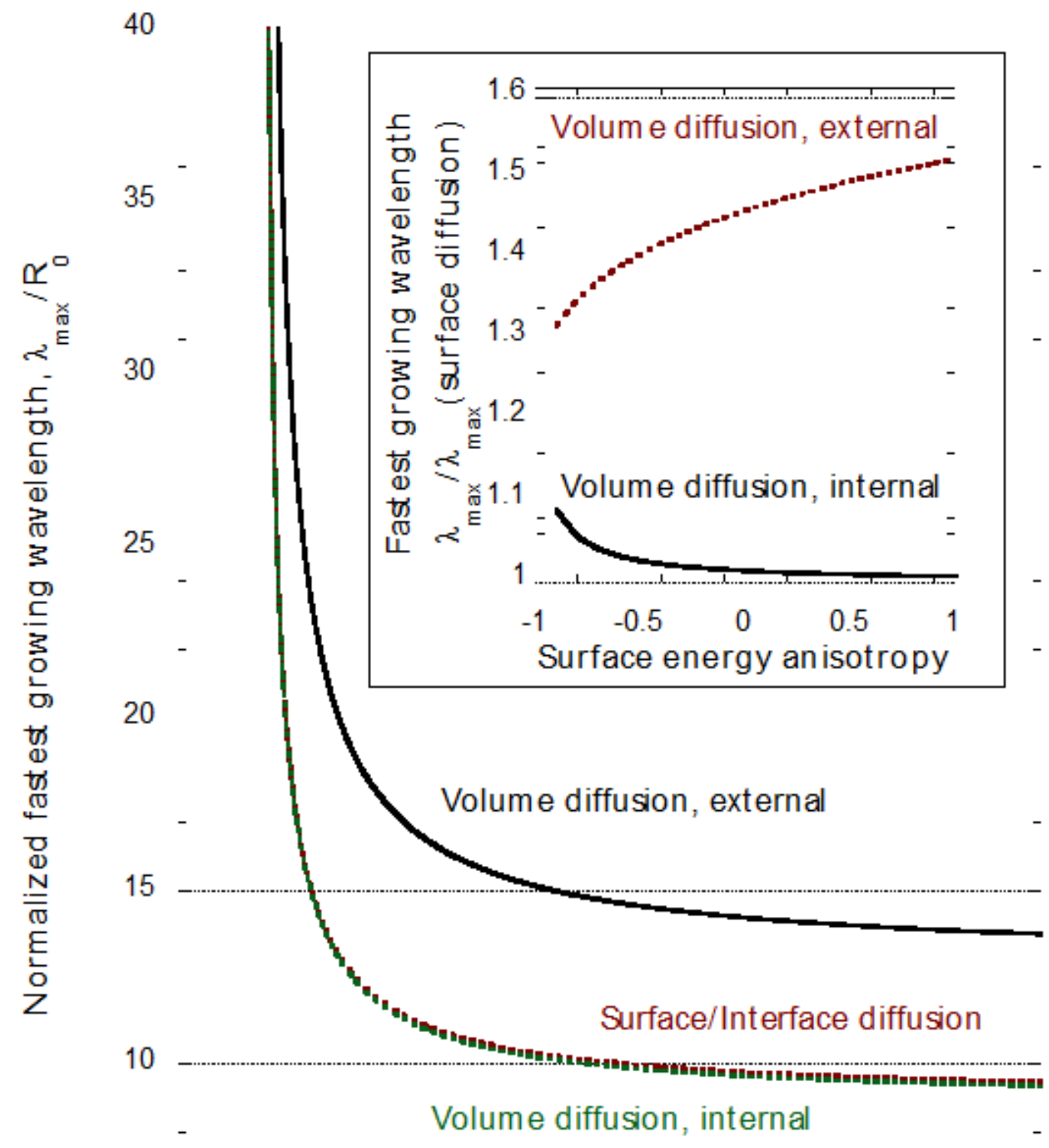

5

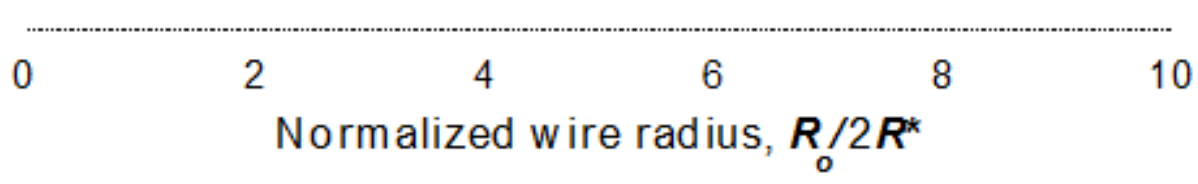

Fig. 3: Plot illustrating the maximal growth wavelength of a perturbation in a wire as a function of its initial radius, $R_{0}$, assuming isotropic interface/surface energy. The difference between interface diffusion and internal volume diffusion is negligible, while the kinetically preferred wavelength for external volume diffusion is clearly larger than the others. The inset represents the effect of surface/interfacial energy anisotropy ( $\left.1+\gamma_{0}^{-1}\left(\partial^{2} \gamma / \partial \phi^{2}\right)_{\phi=0}\right)$ on the maximal wavelength. The difference between interface and internal volume diffusion is greatest for large, negative surface/interfacial energy 
anisotropies.

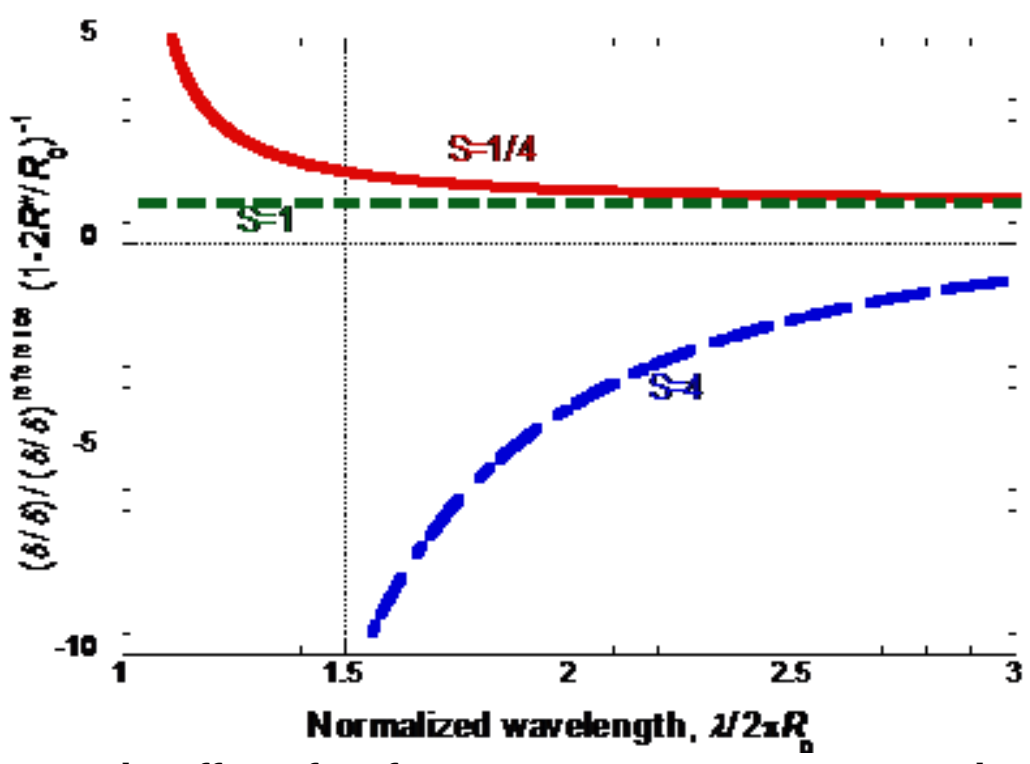

Fig. 4: Plot illustrating the effect of surface-energy anisotropy on normalized amplitude growth rate. The normalized function $(\dot{\delta} / \delta) /(\dot{\delta} / \delta)^{\text {ref }}$ describes how the growth rate is affected by the stabilization factor, S. Consistent with our predictions, breakup is sped up if $\mathrm{S}<1$ and inhibited if

$\mathrm{S}>1$.

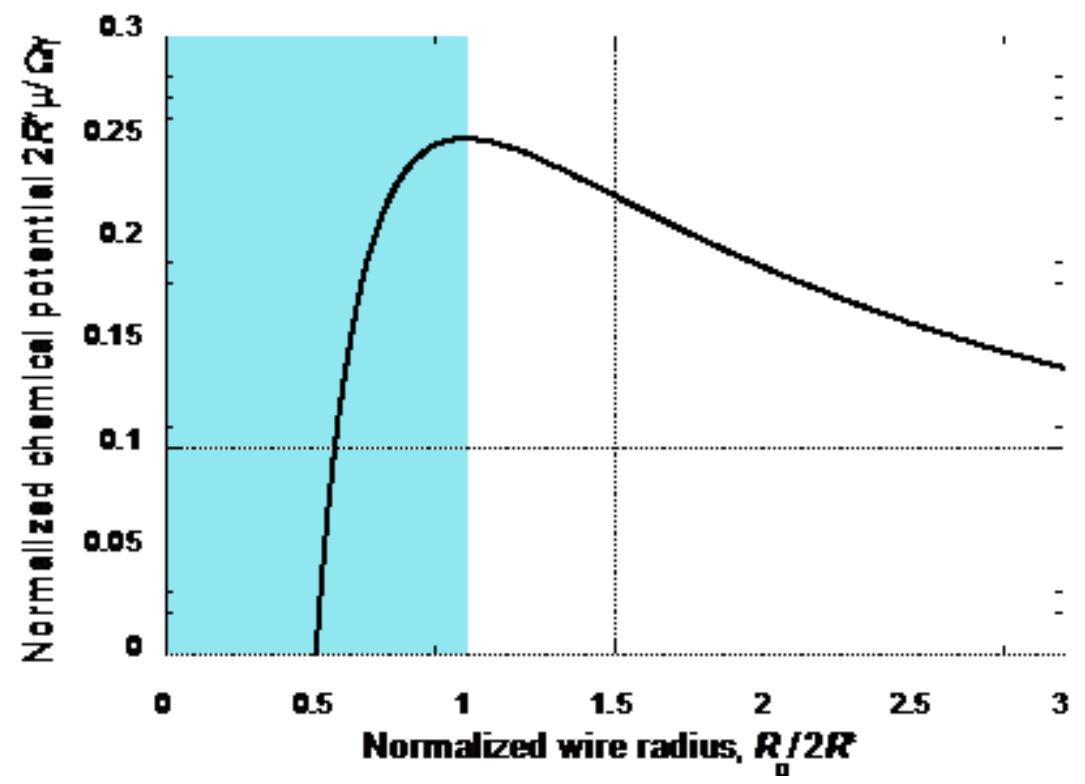

Fig. 5: The dependence of the chemical potential of a wire with radius $R_{0}$. The curve exhibits a maximum if the radius is twice the Frank radius, $R^{*}$. If a wire is smaller than this size (in the shaded area), then its energy would decrease if it shrank. If all wires are smaller than this size, then there is a driving force for all wires to shrink to the same size. 